\title{
CHARACTERISTICS OF SOLAR ACTIVITY AND ITS POSSIBLE RELATIONSHIP WITH EAST ASIAN SUMMER MONSOON
}

\author{
$\mathrm{LI}, \mathrm{X} .{ }^{1}-\mathrm{TU}, \mathrm{S} .^{2, *}$ \\ ${ }^{1}$ Civil Aviation Flight University of China, 618307 Guanghan, China \\ ${ }^{2}$ Faculty of Information Technology, Beijing University of Technology, 100124 Beijing, China \\ *Corresponding author \\ e-mail: sstu@bjut.edu.cn
}

(Received $1^{\text {st }}$ Dec 2018; accepted 20 $0^{\text {th }}$ Feb 2019)

\begin{abstract}
In order to study the variation of solar activity and reveal its relationship with the intensity change of East Asian summer monsoon (EASM), the sunspot time series and EASM intensity index are calculated by using sunspot relative number (SRN) data and sea level barometric data respectively. Then, Morlet wavelet analysis and correlation analysis are used to reveal the interdecadal characteristics of solar activity and EASM changes with their relationship. The results show that after the 1940s, the SRN and the fluctuation amplitude of the EASM index increased gradually, and reached the maximum from the 1950 s to the mid-1960s. The influence of solar activity on the interdecadal variations of EASM is significant, with the same 40 -year and 11-year cycles. The correlation between them is relatively good on the centennial scale, showing a "- + - " correlation with time change.
\end{abstract}

Keywords: sunspot relative number (SRN), wavelet analysis, monsoon index, periodic signal, interdecadal variation

\section{Introduction}

Solar activity is one of the main driving factors of global climate and environmental change. The relationship between solar activity and medium-term and long-term climate changes larger than or equal to 10 years has become a research hotspot in climatology in recent years (Zhao et al., 2012; Gray et al., 2016). Solar activity may affect regional climate in two ways. On the one hand, the abnormal changes of solar radiation affect the weather, climate and ocean thermal state. On the other hand, by stimulating the anomaly of the earth's magnetic field which fills the whole space of the earth, the motion state of the ocean and atmosphere changes, resulting in climate changes including weather scale, interannual scale, interdecadal scale and century scale (Haigh, 1996; Li et al., 2013; Yin et al., 2017). Study results have shown that solar activity is the basic factor affecting the temperature change over 10 years (Hood and Soukharev, 2012; Antico and Torres, 2015), and temperature change can lead to the change of zonal temperature gradient and Hadley circulation, and then affect the long-term change of monsoon ( $\mathrm{Li}$ et al., 2018a, b). There is a strong correlation between solar activity and Asian monsoon activity. Neff et al. (2001) found that there is a strong correlation between the summer monsoon precipitation and the solar activity in the past 3000 years. The intensity of Indian monsoon in the past 1200 years corresponds well with the intensity of solar activity, and they have the same 200-year and 100-year cycles (Agnihtri et al., 2002). The results of Wang et al. (2005) showed that the rotation speed of the earth and the length of sunspot cycle are the main factors that restrict the interdecadal variations of Asian monsoon. The high-frequency variations of the East Asian summer monsoon (EASM) climate are driven by solar activity, and the 10-100-year monsoon climate 
variations are basically consistent with the solar activity cycle (Wu et al., 2006). However, the relationship between solar activity and EASM intensity, which has a significant impact on climate, remains to be studied.

China is a typical monsoon region in the world. The temperature and precipitation of interannual, interdecadal and even intercenturial climate changes in China are affected by the EASM (Hsu et al., 2011; Sun et al., 2015; Ding et al., 2018). Therefore, it is of great importance to study the interdecadal variations of EASM for understanding the causes of interdecadal climate variations of China. In order to further and quantitatively study the multi-time scale changes of monsoon and to have a consistent understanding of the characteristics of strong and weak monsoon, many scholars have established various indices reflecting the strength of EASM based on different data and methods (Guo, 1983; Wang et al., 2001; Sun et al., 2002; Zhang et al., 2003). For example, Guo (1983) defined the EASM index by using the East-West sea-level atmospheric pressure difference, which to a certain extent reflects the difference of sea land thermal power determining the EASM through the difference of land-sea pressure system, and the index has a relatively good effect in reflecting precipitation. These studies show that the period from 1951 to the mid-1960s has strong summer monsoon, after which the summer monsoon weakens, and after 1976, the intensity of summer monsoon weakens again.

It can be seen from the above that due to the lack of data before 1948, the time range of the study was concentrated in the later period, and the time series was short, so the long-term variation trend and interdecadal variation characteristics of EASM intensity were not known. Since only the UK's sea level pressure (SLP) data can be dated back to 1850 , this paper uses the monthly SLP data after 1850 released by the UK's Hadley center to form a long-term EASM index series and study its interdecadal variation characteristics. At the same time, the relationship between solar activity and EASM is still unknown from the above analysis. Therefore, this paper attempts to study the characteristics of solar activity and monsoon intensity change on a centennial scale, to find the relationship between them, and to reveal the possible interaction and influence ways, so as to provide indicative information for the EASM forecast.

\section{Data and methods}

Sea level atmospheric pressure data (HadSLP2) released by the Hadley Center. Time: 1850-2006, monthly average; Space: $5 * 5$ degree grid global coverage. The research scope selected in this paper is " $20-50^{\circ} \mathrm{N}, 110-160^{\circ} \mathrm{E}$ ". This set of data is the second edition released by Hadley Center. It is the longest SLP data at present, and is conducive to calculating the East Asian summer monsoon index for more than 100 years.

The annual average sunspot relative number (SRN) data, published by the Solar Activity Data Analysis Center (SIDC), Institute of solar physics, the Royal Observatory of Belgium, time: 1700-2006.

The main methods used are Morlet wavelet analysis, correlation analysis and Butterworth bandpass filtering.

Morlet wavelet analysis has the characteristics of multi-resolution analysis, and is able to characterize the local characteristics of signals in both time and frequency domains. Wavelet transform decomposes the time series into the time-frequency domain, and then obtains the significant fluctuation patterns of the time series, i.e., the 
periodic change dynamics and the temporal pattern of the periodic change dynamics. In essence, the signal is decomposed by a function's wavelet sequence, which is obtained by scaling and translation of a basic wavelet (wavelet base) or mother wavelet. The scaling and translation are carried out simultaneously in the mother wavelet function. Mother wavelet plays a key role in scale selection and wavelet transform. The applicability of the wavelet function should be fully considered in the selection of the wavelet functions, because the wavelet function used in the wavelet analysis is not unique, i.e., the wavelet functions are diversified. Analyzing the same problem with different wavelet bases will produce different results. At present, the results of signal processing and theoretical results of wavelet analysis are mainly used to evaluate the quality of the wavelet bases, and then the wavelet bases are selected. Morlet wavelet is a complex form of wavelet. It can separate the modulus and phase of the wavelet transform coefficients, where modulus represents the number of components of a certain scale, and phase can be used to study the singularity and instantaneous frequency of signals (Wang et al., 2005).

Correlation analysis method is to calculate the correlation coefficient of any two meteorological elements. It is used to measure the statistical quantity of the relationship between two meteorological elements. The correlation coefficient $r_{x y}$ is the correlation between $n$ time series $x_{i}$ and another time series $y_{i}$. The calculation method is Equation 1.

$$
r_{x y}=\frac{\sum_{i=1}^{n}\left(x_{i}-\bar{x}\right)\left(y_{i}-\bar{y}\right)}{\sqrt{\sum_{i=1}^{n}\left(x_{i}-\bar{x}\right)^{2} \sum_{i=1}^{n}\left(y_{i}-\bar{y}\right)^{2}}}
$$

\section{Variation characteristics of SRN}

Sunspot is considered as the main body of solar activity, and its variation is very regular. It is often used as an important indicator of the intensity of solar activity. In this paper, the interdecadal variations of solar activity are studied by using the SRN data published by the SIDC of the Royal Observatory of Belgium. As can be clearly seen from Figure 1, the SRN has a marked upward trend on the whole, and the fluctuation amplitude of sunspot number in the past three hundred years increased gradually after the 1940s.

\section{Results}

\section{Interdecadal variations of sunspot activity}

In order to study the periodic variation characteristics of solar activity, Morlet wavelet analysis is performed on the SRN series. From the wavelet transform (Fig. 2a), it can be seen that there are obvious quasi-periodic oscillations at the levels of 80, 100, 40-50 and 11 years in the variation of the intensity of sunspot activity. At the 50-year level, the SRN has weakened since the 1960s, and the wavelet values and amplitudes have decreased significantly. The changes of 50-year cycles gradually weakened and disappeared, and turn into 40-year cycles. At the 11-year level: from the 1940s to the end of the 1980s, solar activity changes most dramatically. From 1950s to mid-1960s, 
the solar activity is the strongest in more than 300 years, which corresponded to the positive center of 180,100, 40 and 11-year cycle changes. Secondly, from the 1970s to the 1990s, and from the 1930s to the 1950s, there are also two periods of strong solar activity. Since the solar cycle gradually changes from 50 years to 40 years around 1850 , the wavelet variance series of 1700-1850 and 1851-2006 are analyzed respectively. From 1700 to 1850 (Fig. 2b), the most prominent period of solar activity is about 100 years, followed by 11 years and 50 years, and finally by 180 years. The 40 -year cycle does not show up in this period. From 1851 to 2006 (Fig. 2c), the 11-year cycle becomes the most prominent cycle of solar activity, followed by the 100 -year cycle, and then the 180-year cycle. The 50-year cycle no longer existed in this period, but a weaker cycle appeared in the 40-year scale. Han and Han $(2001,2002)$ pointed out that many periodic components of solar activity are not fixed, but have varying degrees of uncertainty and time-varying characteristics. That is, the period length, amplitude and phase change with time, which is consistent with the results of this paper.

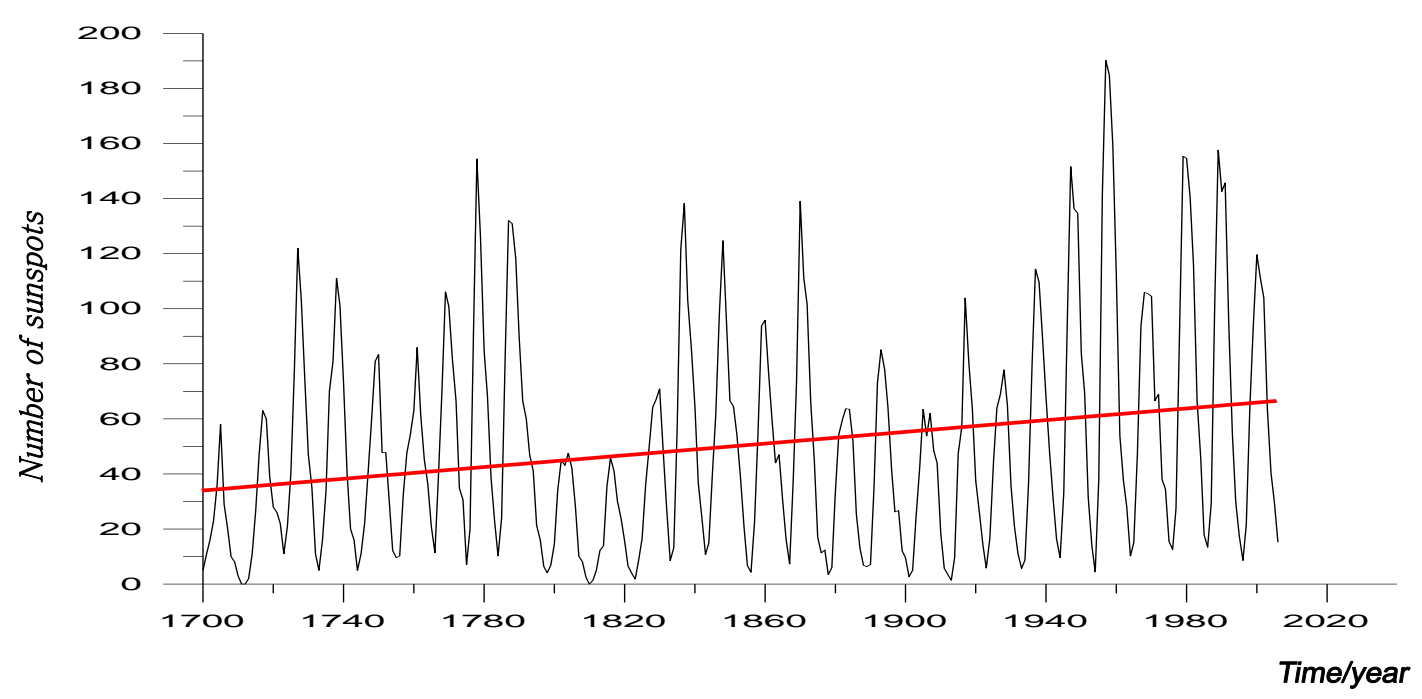

Figure 1. Time series curve of SRN (Oblique line is trend line)

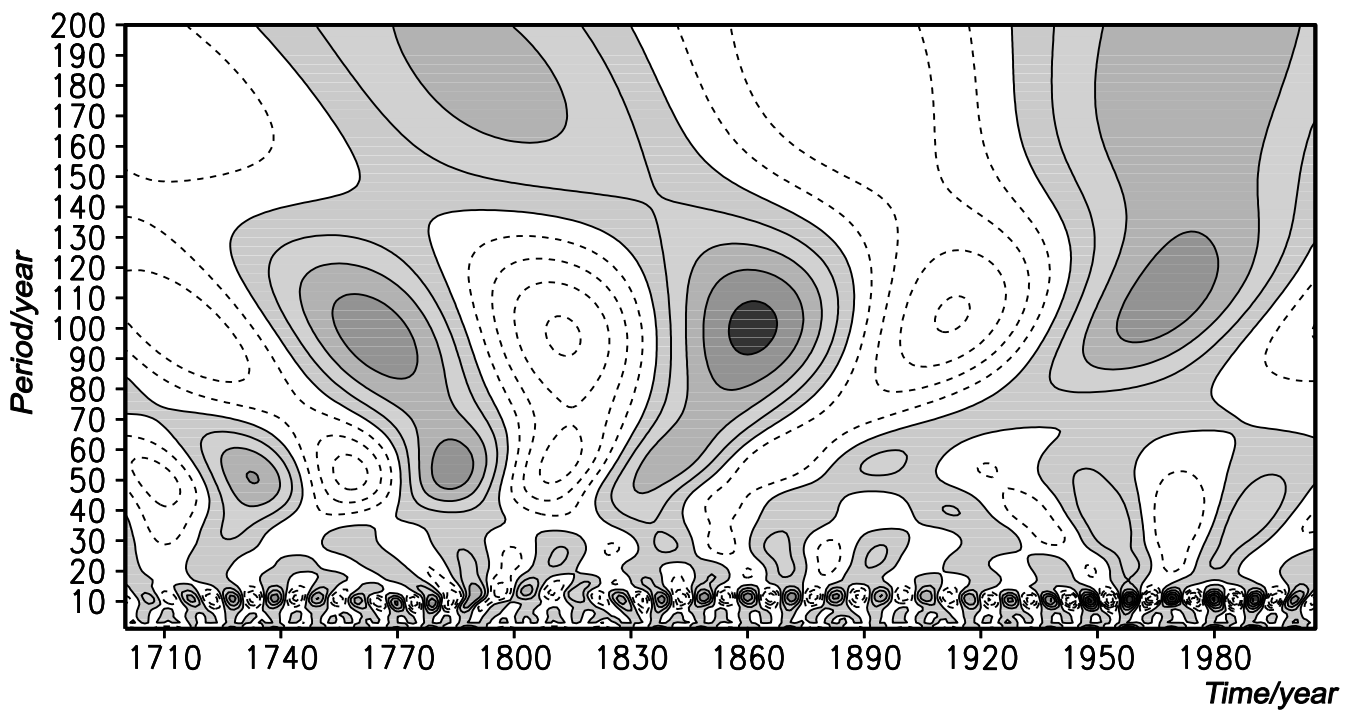

$\mathbf{a}$ 


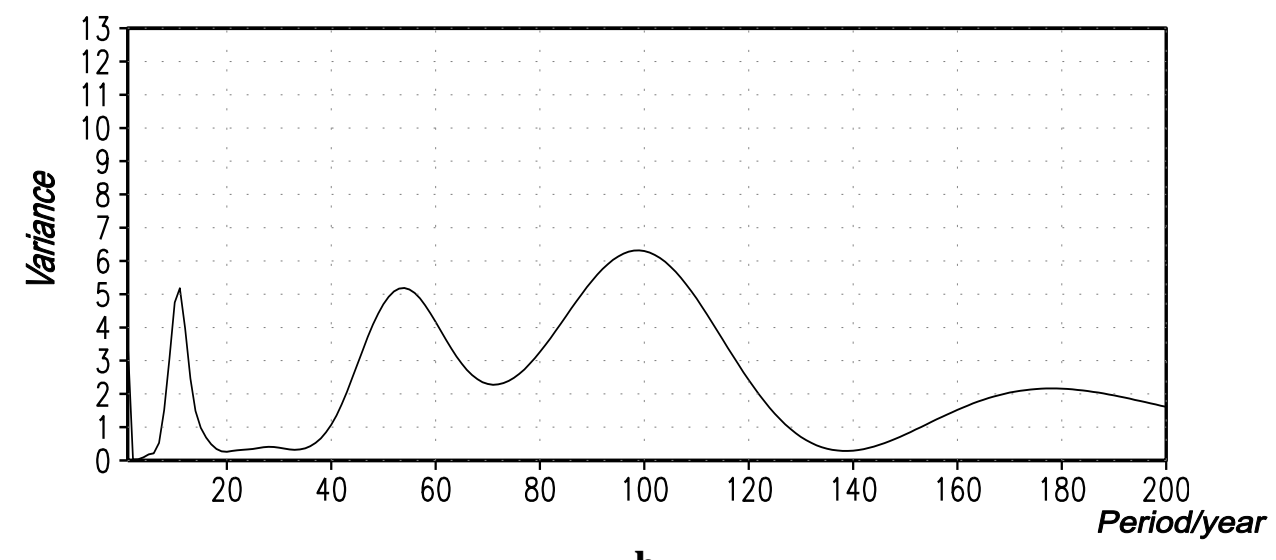

b

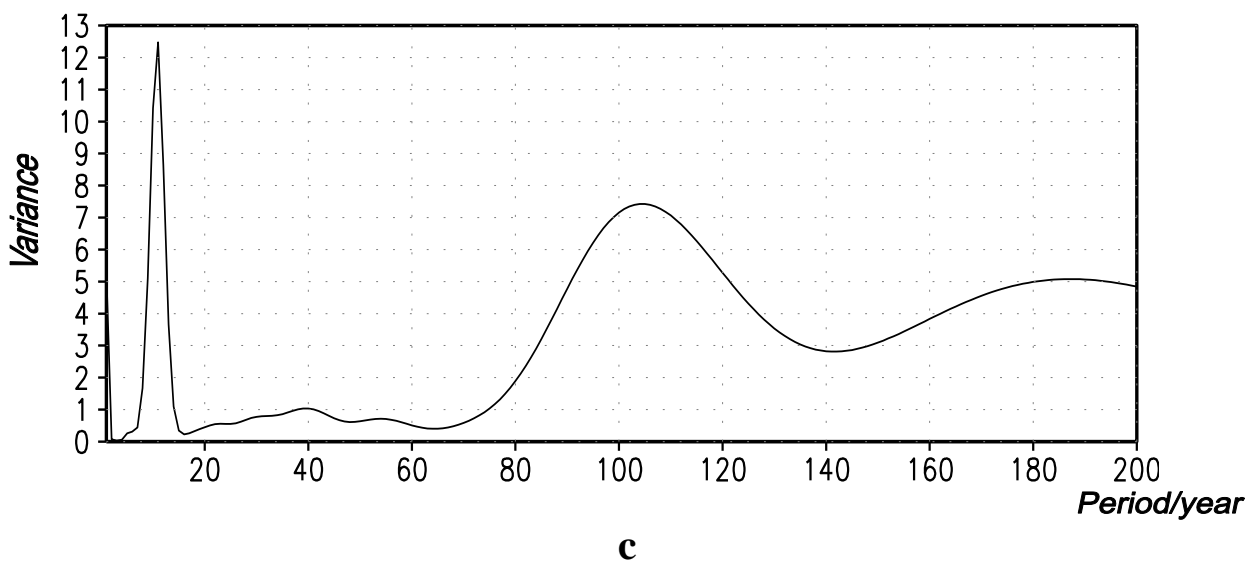

Figure 2. a Wavelet transform map of SRN in 1700-2006. $\boldsymbol{b}$ Wavelet variance sequence of SRN in 1700-1850. $c$ Wavelet variance sequence of SRN in 1850-2006

\section{Interdecadal variation characteristics of EASM}

\section{Intensity variation of EASM}

In order to reveal the interdecadal variability of the EASM and its relationship with solar activity, the EASM index is selected and compared. Since the monsoon system is affected by the high and low latitudes systems at the same time, and there are interactions and constraints among various factors, each monsoon index can only describe one aspect of the characteristics of the monsoon better. At present, there is still no index that can describe the characteristics of monsoon variations in all aspects comprehensively. Considering the long-term nature of the time scale of the data, this paper chooses the method of calculating the EASM Index (EASMI) defined by Guo (1983), The strength of EASM is represented by the cumulative sum of $\triangle \mathrm{P} \leq-5 \mathrm{hPa}$ at each 10 latitudes $\left(\sum \triangle \mathrm{P}\right)$ through the $110-160^{\circ} \mathrm{E}$ pressure difference $(\triangle \mathrm{P})$, between June and August $20-50^{\circ} \mathrm{N}$. That is:

$$
I_{\mathrm{EASM}}=\Sigma \square P\left[S L P\left(20-50^{\circ} N, 110-160^{\circ} E\right)\right]
$$

Figure 3 shows the East Asian summer monsoon index series calculated by Equation 2 and normalized. As can be clearly seen from Figure 3, the EASM 
experienced four stages of strong-weak-strong-weak change in 1850-2006, which is non-linear and a long-time scale fluctuation. From the 1950s to the mid-1960s, the intensity of the EASM increased in general, and it reached its strongest stage in the $1950 \mathrm{~s}$ and 1960s. This stage is also the strongest stage of solar activity analyzed in Figure $2 a$, which has been decreasing since the mid-1960s. The intensity of EASM changed abruptly in the mid-1960s and experienced a transition from the strongest to the weakest. Weak summer monsoon lasted until 2006, it reached its weakest stage after the mid-1970s. It is noteworthy that the fluctuation amplitude of the EASM increased gradually after the 1940s. Compared with the time series curve of SRN in Figure 1, it can be seen that the fluctuation amplitude of SRN has also increased gradually since 1940s. In conclusion, it can be concluded that the intensity of sunspot activity oscillation may be one of the reasons for the intensity oscillation of EASM.

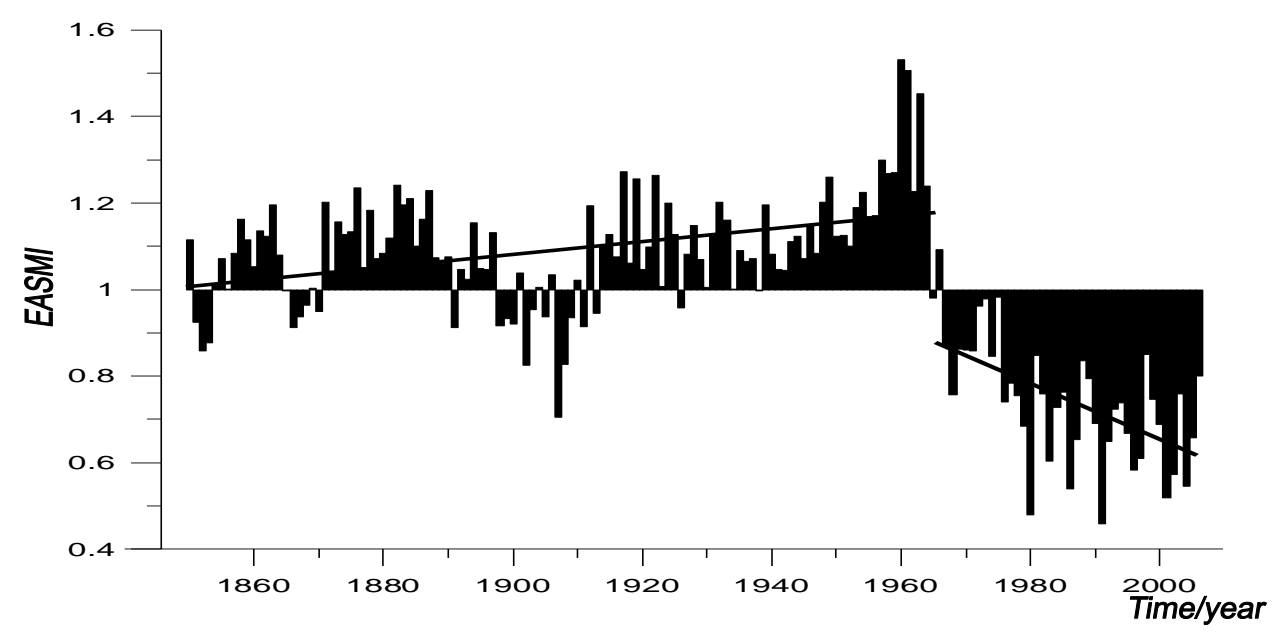

Figure 3. EASM Index series (Dimensionless, oblique line is trend line)

\section{Interdecadal variation period analysis of EASM}

From the previous analysis, we can see that the solar activity shows some interdecadal periodic changes. In order to study the possible relationship between solar activity and EASM, the wavelet transform and wavelet variance maps of the abovementioned EASM indices are presented, and their periodic variation characteristics are analyzed.

As can be seen from Figure $4 a$, the EASM in 1850-2006 has obvious quasi-periodic oscillations in the levels of 80,40, 20 and 10-13 years. The 40-year cycle has a decreasing trend since the 1950s; the 20-year cycle has increased since the 20th century, with an increasing trend of amplitude; and the 11-year cycle has also gradually increased since the 1950s. It can also be seen that the period from 1950s to mid-1960s was the strongest period of EASM. The density, numerical value and time level of the wavelet transform curve in this period was the largest in 157 years, and then followed by the weakest period of EASM, which is consistent with the previous analysis results. From the wavelet variance figure of Figure $4 b$, it can be seen that the main interdecadal period of the EASM is about 80 years, followed by about 40 years. In addition, there is a weak period of about 11 years. The period of about 20 years is not shown in the wavelet variance map because of the weak signal. 

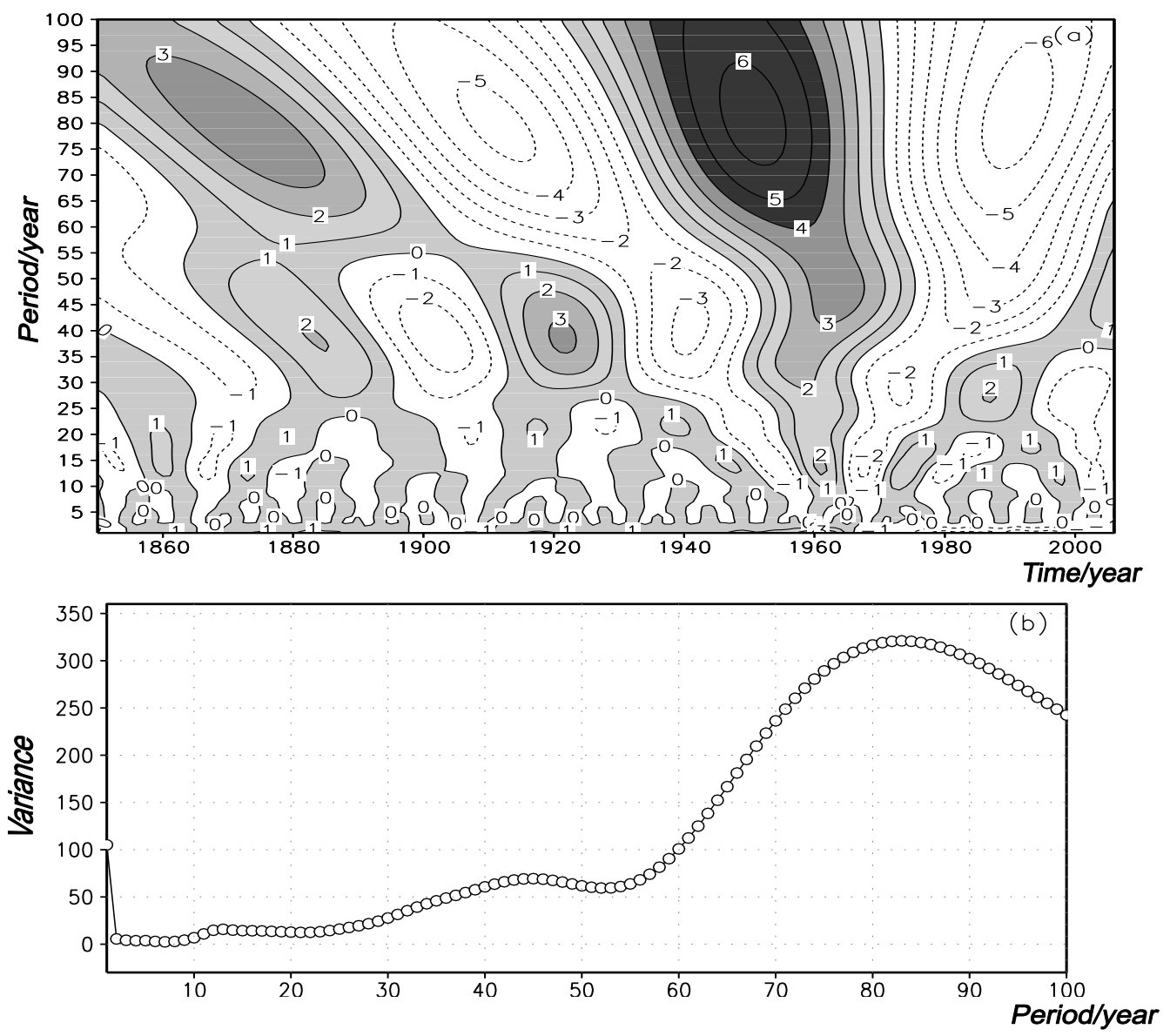

Figure 4. a Wavelet transform map of the EASM index. $\boldsymbol{b}$ Wavelet variance map of EASM index. (In order to clearly show the period, the average wavelet variance in the figure is expanded by 10 times)

\section{Relationship between EASM and solar activity}

The above analysis shows that the solar activity has the same 40-year and 11-year cycle as the EASM intensity change. EASM and solar activity reached the strongest in all years from 1950s to mid-1960s. Therefore, the influence of solar activity on the interdecadal variation of the EASM intensity does exist. Comparing the EASM index and sunspot number series from 1850 to 2006, it is found that before the mid-1960s, both of them had an increasing trend, and then they changed in the opposite direction. In order to discuss the interdecadal correlation between the two, the 157 EASM index is averaged by a nine-year moving window, and the interannual variation information is filtered out. Then the sunspot number is averaged by an 11-year moving window, eliminating its inherent 11-year cycle. In this way, the interdecadal relationship between EASM intensity and solar activity can be better reflected. The smoothed values are shown in Figure 5. The variations of the EASM and sunspot activity are not always consistent. The EASM and sunspot number curves show obvious negative correlation changes before the 20th century, then positive correlation changes from the beginning of 20th century to the mid-1960s, and then negative.

Kilcik et al. (2007) studied the influence of solar activity on climate from 1940 to 1984, and concluded that there was a positive correlation between solar activity 
parameters and terrestrial atmospheric temperature before 1966, but a negative correlation after 1966, which is consistent with the results of this study. The correlation coefficients between the moving averaged EASM index and the SRN are calculated. The results show that the correlation coefficients are -0.845 before $1910,0.723$ between 1911 and 1965 , and -0.489 after 1966, all exceeding the reliability test of 0.001 . Based on the wavelet analysis of sunspot activity, it is found that the correlation between EASM and sunspot activity is affected by the 100-year cycle of solar activity. When the sunspots number decreases from the peak in 1850 to the valley in 1910 on a centennial scale (Fig. 2a), there is a negative correlation between the sunspots number and EASM index, i.e., EASM is strong in the year with weak solar activity, while it is weak in the year with strong solar activity. When the sunspot number transits from the valley value in 1910 to the peak value in 1965, EASM index is positively correlated with solar activity, i.e., EASM is strong when solar activity is strong, and is weak when solar activity is weak. Similarly, since 1965, solar activity has shifted from peak to valley on a centennial scale, and the relationship between them has changed again. It can be concluded that solar activity not only has a strong influence on the intensity change of EASM at interdecadal scale, but also is an important factor that can not be ignored in influencing the intensity change of summer monsoon at centennial scale.

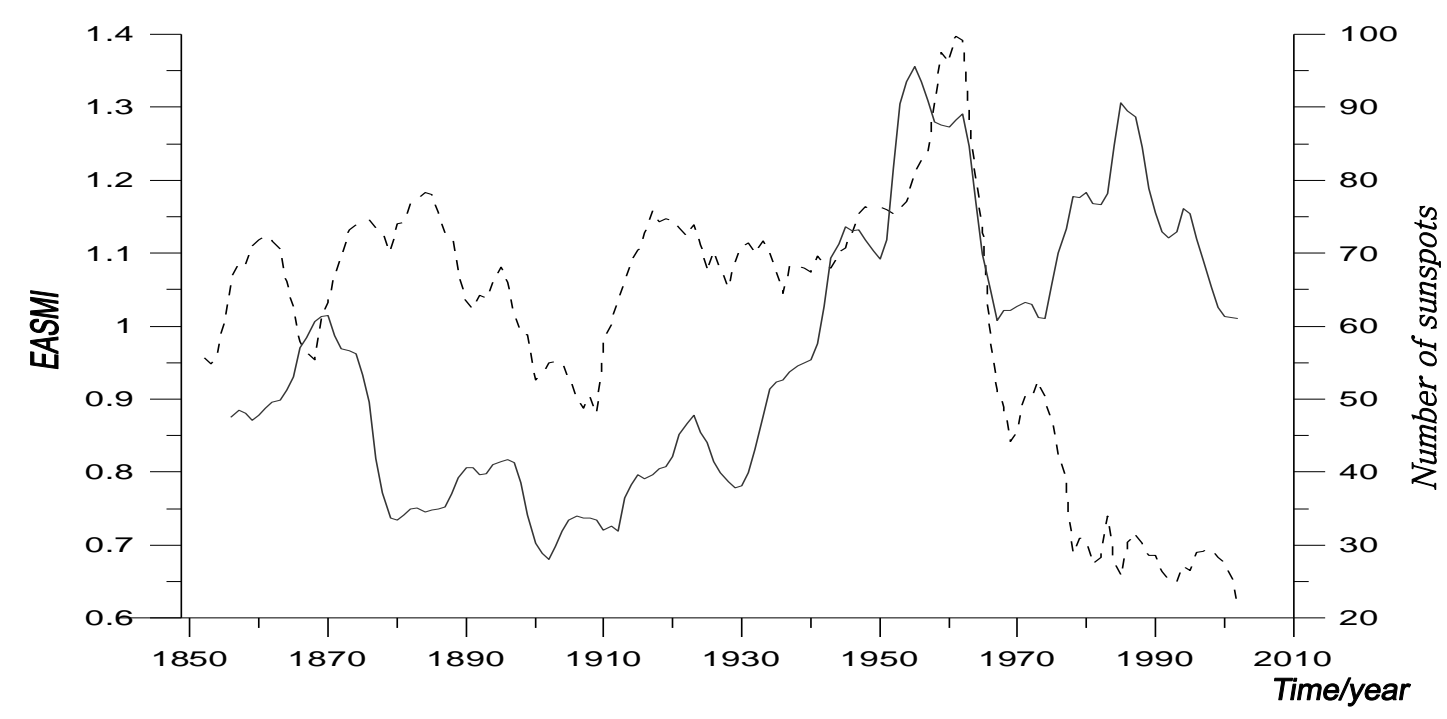

Figure 5. Moving average curve of SRN (real line) and EASM index (dotted line)

\section{Analysis of solar activities affecting interdecadal variation of EASM intensity}

The interdecadal variations of solar activity and EASM intensity are well correlated. Based on the previous analysis, the influence information of solar activity in the EASM index series is extracted and analyzed step by step in this section. When the information is separated from the concealed state, the relationship between them can be revealed, which provides a new basis for the interdecadal variation of the EASM.

The analysis of the EASM intensity index shows that in the mid-1960s, EASM changed dramatically. Before the mid-1960s, EASM generally strengthened. After reaching the strongest point in the mid-1960s, it suddenly changed and weakened, and then entered the period of weak summer monsoon. The trend line in Figure 3 shows the trend changes before and after the sudden change of summer monsoon. 
When $i=1,2,3 \ldots \ldots 117, l_{1}(i)$ represents the trend state before the sudden change;

When $i=118,119 \ldots . . .157, l_{2}(i)$ represents the trend state after the sudden change;

The general trend changes:

$$
l(i)=l_{1}(i)+l_{2}(i) \quad i=1,2,3 \ldots \ldots 157
$$

By subtracting the climatic trend $l(i)$ in Equation 3 from the original summer monsoon index series $I_{s m}(i)$, the summer monsoon index series without climatic trend, $Y_{s m}(i)$, can be obtained.

$$
Y_{s m}(i)=I_{s m}(i)-l(i)
$$

The mean square deviation of the original summer monsoon index series $I_{s m}(i)$ is obtained.

$$
S=\sqrt{\frac{1}{n} \sum_{i=1}^{n}\left(I_{s m}(i)-\bar{I}_{s m}{ }^{2}\right.}=0.204
$$

where $\overline{I_{s m}}$ is the average EASM index for 157 years during 1850-2006. Similarly, the mean square deviation of EASM index series after the trend change is filtered out according to Equation 5:

$$
S_{1}=\sqrt{\frac{1}{n} \sum_{i=1}^{n}\left(Y_{s m}(i)-{\overline{Y_{s m}}}^{2}\right.}=0.129
$$

where $\overline{Y_{s m}}$ is the average of EASM index series $Y_{s m}(i)$ defined in Equation 4 after filtering out trend change during 1850-2006. Then the variance contribution of trend change to the summer monsoon index series is as follows:

$$
\begin{aligned}
S S & =\left[\left(S-S_{1}\right) / S\right] \times 100 \% \\
& =[(0.204-0.129) / 0.204] \times 100 \%=37 \%
\end{aligned}
$$

In the formula, $S$ and $S_{1}$ are calculated by Equations 5 and 6 respectively. The variance contribution of the trend is $37 \%$, i.e., the increase of EASM intensity before the mid1960s and the subsequent abrupt decrease are the basic characteristics of the change in the whole 157 years.

The wavelet analysis of EASM index and SRN shows that there exists the same 40year and 11-year cycle between EASM and sunspot activity. The 40-year periodic component series of EASM $l_{40}(i)$ is obtained by band-pass filtering. By subtracting 40year cycle components from the original summer monsoon index series, the index series without 40 -year cycle $Y_{s m}(i)$ can be obtained. The mean square deviation of $Y_{s m}(i)$ 
obtained from Equation 5 is 0.159 . According to Equation 7, the contribution of 40-year cycle change to the variance of summer monsoon variability is $22 \%$.

It can be seen that the 40-year cycle is one of the main cycles of the EASM. Using the same method, the variance contribution rate of 11 -year cycle is $14 \%$, and that of $80-$ year cycle is $25 \%$, which ranks only second to trend change.

The 11-year and 40-year cycles play an important role in interdecadal variations of the intensity of the East Asian summer monsoon. In order to further understand the influence of 11-year and 40-year cycles of solar activity on the variation of EASM, the wavelet component curves of the EASM index and SRN in the period of 40 and 11 years from 1850 to 2006 are plotted in Figure 6.

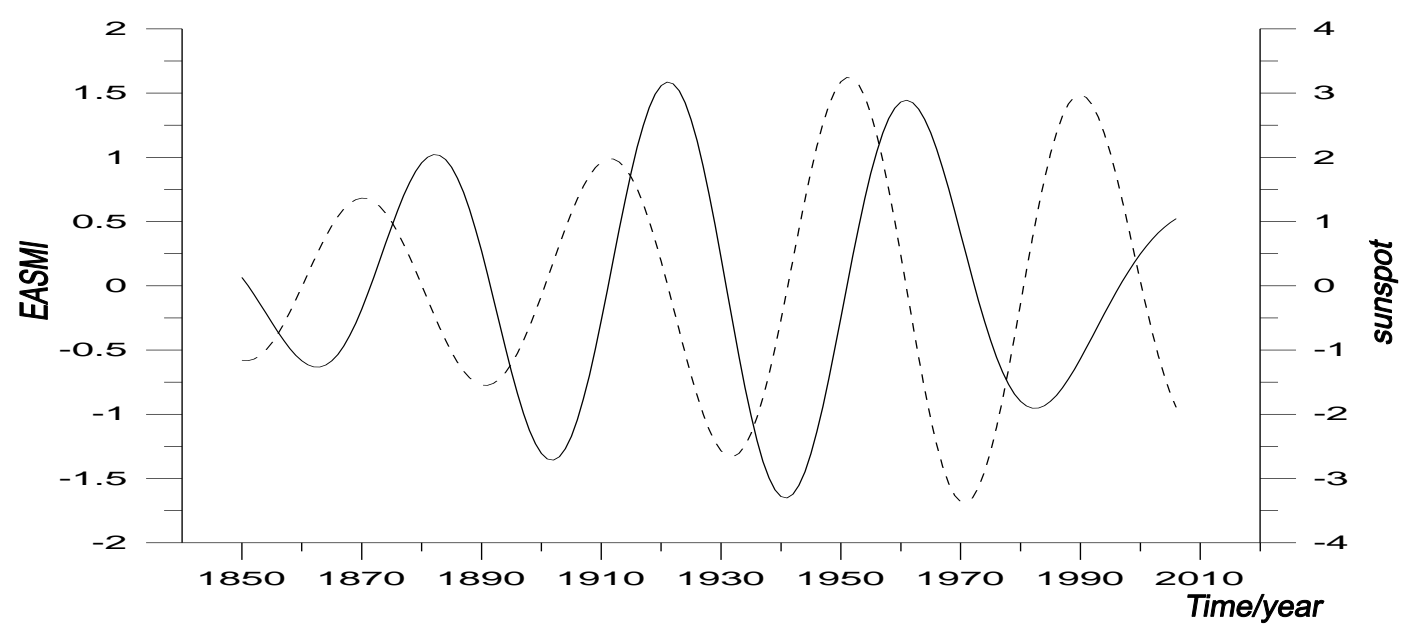

$\mathbf{a}$

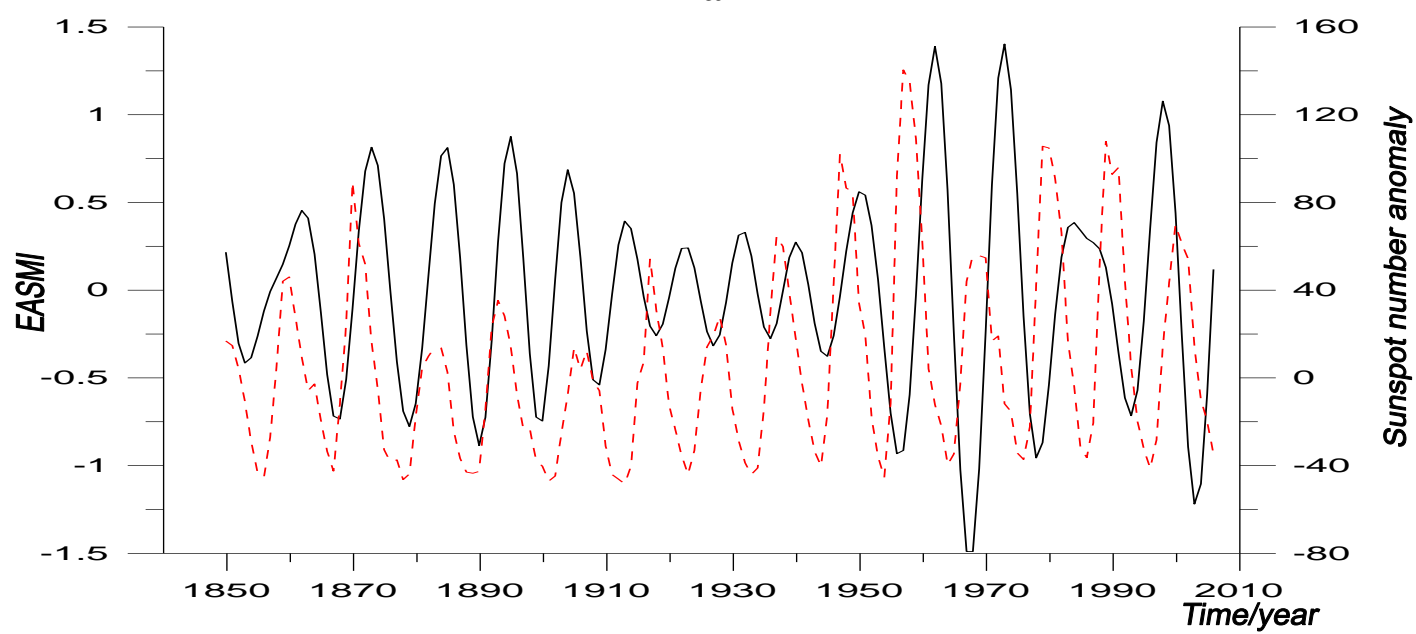

b

Figure 6. a 40-year wavelet periodic component curve of EASM index and SRN. $\boldsymbol{b} 11$-year wavelet periodic components of EASM index and anomaly values of SRN. (Real line and left coordinate are EASM index, while dotted line and right coordinate are $S R N)$

As can be seen from the figure, there is a good positive correlation between the variation of EASM and the 40-year cycle of sunspot (Fig. 6a). The same is true for the magnitude. When the magnitude of sunspot variations increases, the magnitude of EASM variations also increases, and the sunspot number is about 10 years ahead of the 
summer monsoon. In the time scale of 11-year cycle (Fig. 6b), the variation curves of the two are generally similar. Before 1910, the variation of sunspot number is isotopic. The variation of sunspot number is 1-3 years ahead of that of EASM index. From 1910 to 1940 , the two gradually changed into inverse phases, and the variation ranges were gentle. After 1940, the two phases gradually changed into the same phase. The magnitudes of the two increased obviously, but the magnitude of the EASM lagged behind the solar activity. The above analysis shows that the influence scale of sunspot activity on EASM variations is from 11 to 40 years, i.e., solar activity plays a decisive role in EASM variations on the "decadal" time scale.

\section{Discussion}

In this paper, the cycle analysis of the SRN and the EASM index variations shows that there is a close relationship between them: the fluctuation amplitude of the two shows a similar trend, with the same change period and strong correlation, and the variations of the EASM lag behind the changes of the SRN. The EASM seriously affects the weather and climate in China and East Asia. Therefore, it is of practical significance to analyze the solar activity to predict the intensity of the summer monsoon and then forecast the weather or climate conditions. At present, most studies focus on the characteristics of solar activity itself (Baldwin and Dunkerton, 2005; Frame and Gray, 2010) and the influence of solar activity on precipitation, temperature and other climatic factors (Maliniemi et al., 2014; Ge et al., 2016). Since some weather and climate systems, such as monsoons, directly influence precipitation. It is also beneficial to reveal the physical mechanisms of the sun affecting climate. Studies have yielded some useful results from this very point (Kerr, 2005; Verschuren et al., 2009), but there has been a lack of research on the East Asian monsoon. Because local climate systems vary from region to region, this paper is of certain value for the relevant research of EASM.

However, the effect of solar activity on interdecadal variations of EASM, and the law of solar activity affecting the variations of EASM are very complex, non-linear in different periods and time scales. Many problems still exist and need to be solved. Small changes in solar radiation over the 10-100-year scale can neither directly cause significant heating changes in the Qinghai-Tibet Plateau, nor affect the EASM. However, solar activity may affect the summer monsoon through atmospheric or oceanic circulation (Zhou and Chen, 2014). At present, the mechanism of solar activity changes affecting East Asian monsoon is not clear. More factors, such as sunspot magnetic field and total solar radiation (Qi et al., 2015), need to be considered to comprehensively study the ways and mechanisms of solar activities affecting EASM through atmospheric ocean circulation and ground radiation, etc.

\section{Conclusion}

In this paper, the main characteristics and main periodic variations of sunspot activity on a centennial scale are analyzed. The interdecadal variations of the EASM and their correlation with sunspot activity are discussed. Morlet wavelet technique is used to discuss them, revealing their common periods and analyzing them separately. The real change of climate factors is the combination of all effective signals and noise signals, i.e., the superposition of different periodic signals. Although the signal obtained by this 
method is only one of its "components", the change of "original" information can be basically mastered by clarifying the change law of these basic periods. Therefore, it is significant to determine the interaction between these basic cycles for the analysis and prediction of interdecadal variations of EASM. The following conclusions can be drawn from this study:

From 1700 to 2006, SRN showed an obvious upward trend, and its fluctuation amplitude increased gradually after 1940s. On the centennial scale, before the 1960s, the sunspots number had significant 11-year, 50-year, 100-year and 180-year cycles, and then 50-year cycles changed to 40-year cycles.

There is a strong correlation between solar activity and EASM intensity variations on the centennial scale, showing a " + - " correlation with time. The intensity of sunspot activity oscillation is one of the reasons for the intensity oscillation of EASM. Both of them reached their maximum from 1950s to mid-1960s, and they have the same 40-year and 11-year cycles.

The influence scale of sunspot activity on EASM variations ranges from 11 to 40 years. That is to say, solar activity plays a decisive role in EASM variations on the "decadal" time scale. The correlation between sunspot activity and EASM variations is regulated by solar activity on the centennial scale.

Acknowledgements. This work was supported in part by the China Civil Aviation Flight Technology and Flight Safety Research Base Open Fund Project (F2016KF02).

\section{REFERENCES}

[1] Agnihtri, R., Dutta, K., Bhushan, R. et al. (2002): Evidence for solar forcing on the Indian monsoon during the last millennium. - Earth and Planetary Science Letters 198: 521-527.

[2] Antico, A., Torres, M. E. (2015): Evidence of a decadal solar signal in the Amazon River: 1903 to 2013. - Geophysical Research Letters 42(24): 10,782-10,787.

[3] Baldwin, M. P., Dunkerton, T. J. (2005): The solar cycle and stratosphere-troposphere dynamical coupling. - Journal of Atmospheric and Solar-Terrestrial Physics 67(1-2): 7182 .

[4] Ding, Y., Si, D., Liu, Y. et al. (2018): On the characteristics, driving forces and interdecadal variability of the East Asian summer monsoon. - Chinese Journal of Atmospheric Sciences 42(3): 533-558.

[5] Frame, T. H., Gray, L. J. (2010): The 11-yr solar cycle in ERA-40 data: an update to 2008. - Journal of Climate 23(8): 2213-2222.

[6] Ge, Q., Liu, L., Zheng, J. et al. (2016): Spatial patterns of drought/flood over eastern China in the periods of anomalous solar activity during the past millennium. - Acta Geographica Sinica 71(5): 707-717.

[7] Gray, L., Woollings, T., Andrews, M. et al. (2016): Eleven-year solar cycle signal in the NAO and Atlantic/European blocking. - Quarterly Journal of the Royal Meteorological Society 142(698): 1890-1903.

[8] Guo, Q. (1983): The summer monsoon intensity index in East Asia and its variation. Acta Geographica Sinica 38(3): 207-217.

[9] Haigh, J. D. (1996): The impact of solar variability on climate. - Science 272(5264): 981984.

[10] Han, Y., Han, Y. (2001): Wavelet analysis on changes of relative sunspot number. Chinese Science Bulletin 46(24): 2031-2034. 
[11] Han, Y., Han, Y. (2002): The relative sunspot number variability over a period of about 5 months. - Chinese Science Bulletin 47(20): 1535-1539.

[12] Hood, L. L., Soukharev, B. E. (2012): The lower-stratospheric response to 11-yr solar forcing: Coupling to the troposphere-ocean response. - Journal of the Atmospheric Sciences 69(6): 1841-1864.

[13] Hsu, P., Li, T., Wang, B. (2011): Trends in global monsoon area and precipitation over the past 30 years. - Geophysical Research Letters 38(12): 8701-8710.

[14] Kerr, R. A. (2005): Changes in the sun may sway the tropical monsoon. - Science 308: 787.

[15] Kilcik, A., Okten, A., Aln, G. (2007): Does the sun affect the earth's climate? - J. Climate 20(3): 24-33.

[16] Li, K. J., Shi, X. J., Xie, J. L. et al. (2013): Solar-cycle-related variation of solar differential rotation. - Monthly Notices of the Royal Astronomical Society 433(1): 521527.

[17] Li, X., Tu, S., Tang, Y. et al. (2018a): Correlation analysis of East Asian summer monsoon and subsurface ocean temperature from the Pacific Ocean to the Indian Ocean in the tropics. - Applied Ecology and Environmental Research 16(1): 81-96.

[18] Li, X., Tu, S., Kang, X. et al. (2018b): The coupling relationship between tropical upper sea temperature field and East Asian summer monsoon. - Applied Ecology and Environmental Research 16(5): 7155-7170.

[19] Maliniemi, V., Asikainen, T., Mursula, K. (2014): Spatial distribution of Northern Hemisphere winter temperatures during different phases of the solar cycle. - Journal of Geophysical Research: Atmospheres 119(16): 9752-9764.

[20] Neff, U., Bums, S. I., Mangini, A. et al. (2001): Strong coherence between solar variability and the monsoon in Oman between 9 and 6 kyr ago. - Nature 411(6835): 290293.

[21] Qi, J., Zhang, P., Qiu, H. et al. (2015): Analysis of total solar irradiance observed by FY3C Solar Irradiance Monitor-II. - Chinese Science Bulletin 60(25): 2447-2454.

[22] Sun, W., Liu, J., Wang, Z. et al. (2015): Modeling study on the characteristics and causes of East Asian summer monsoon precipitation on centennial time scale over the past 2000 years. - Advances in Earth Science 30(7): 780-790.

[23] Sun, X., Chen, L., He, J. (2002): Index of land-sea thermal difference and its relation to interannual variation of summer circulation and rainfall over East Asian. - Acta Meteorologica Sinica 60(2): 164-172.

[24] Verschuren, D., Damste, J. S., Moernaut, J. et al. (2009): Half-precessional dynamics of monsoon rainfall near the East African Equator. - Nature 462: 637-641.

[25] Wang, B., Wu, R., Lau, K. M. (2001): Interannual variability of the Asian Summer Monsoon: Contrasts between the Indian and the western North Pacific East Asian monsoons. - J. Climate 14: 4073-4090.

[26] Wang, W., Ding, J., Li, Y. (2005): Hydrological Wavelet Analysis. - Chemical Industry Publishing, Beijing, pp. 115-141.

[27] Wang, Y., Cheng, H., Edwards, R. L. et al. (2005): The Holocene Asian monsoon: links to solar changes and North Atlantic climate. - Science 308(5723): 854-857.

[28] Wu, J., Shao, X., Kong, X. et al. (2006): The trace of the solar activity in Nanjing during the Last Glacial Maximum. - Chinese Science Bulletin 51(4): 431-435.

[29] Yin, Z., Liu, D., Pang, C. et al. (2017): The centennial to millennial-scale cycles analysis of holocene climatic variability and solar activity. - Periodical of Ocean University of China 47(7): 112-120.

[30] Zhang, Q., Tao, S., Chen, L. (2003): The inter-annual variability of East Asian summer monsoon indices and its association with the pattern of general circulation over East Asia. - Journal of Meteorological Research 61(4): 559-568.

[31] Zhao, L., Wang, J. S., Zhao, H. J. (2012): Solar cycle on decadal variability in monsoon precipitation over China. - J. Meteor. Soc. Japan 90(1): 1-9. 
[32] Zhou, Q., Chen, W. (2014): Impact of the 11-year solar cycle on the relationship between the East Asian winter monsoon and the following summer monsoon and the related processes. - Climatic and Environmental Research 19(4): 486-496. 